\title{
Genotype-Phenotype Correlation in Long-Term Cohort of Japanese Patients with Moyamoya Disease
}

\author{
Shunsuke Nomura ${ }^{a}$ b Koji Yamaguchi ${ }^{a}$ Hiroyuki Akagawa ${ }^{b}$ \\ Akitsugu Kawashima $^{c}$ Yosuke Moteki $^{\text {a }}$ Tatsuya Ishikawa $^{a}$ Yasuo Aihara ${ }^{a}$ \\ Taiichi Saito $^{a}$ Yoshikazu Okada ${ }^{a}$ Takakazu Kawamata ${ }^{a}$

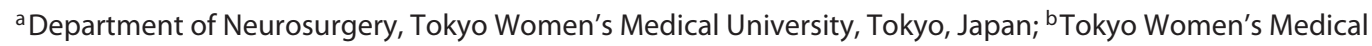 \\ University Institute for Integrated Medical Sciences (TIIMS), Tokyo, Japan; ' Department of Neurosurgery, Tokyo \\ Women's Medical University Yachiyo Medical Center, Tokyo, Japan
}

\section{Keywords}

Genotype-phenotype correlation · Long-term outcome ·

Moyamoya disease $\cdot$ RNF213

\begin{abstract}
Background: Homozygosity of this p.R4810K founder variant of RNF213 moyamoya disease (MMD) susceptibility gene is known to influence the severity of the clinical disease phenotype at disease onset. However, the association between this genotype and long-term clinical manifestations has remained unclear. Objectives: The principal goal of this study was to investigate whether and how the p.R4810K variant of RNF213 influences the long-term phenotype in Japanese patients with MMD. Method: This retrospective cohort study included 94 Japanese patients with MMD who underwent direct or combined bypass for revascularization with the p.R4810K genotype determined in our hospital. The following phenotypic parameters were analyzed at disease onset and over a long-term period: age and initial presentation at onset, recurrent stroke after initial revascularization, and final modified Rankin Scale. Results: The p.R4810K genotype was significantly associated with the phenotype at onset, especially in younger patients. Over a median follow-up period
\end{abstract}

\section{KARGER}

(C) 2019 S. Karger AG, Basel

E-Mail karger@karger.com

www.karger.com/ced of 100 months, recurrent stroke occurred in 6 out of 94 patients: none out of 5 patients with the homozygous variant, 5 out of 64 with the heterozygous variant, and 1 out of 25 in the wild-type group. There were no significant differences among the genotypes. In particular, recurrent cerebral hemorrhage occurred in 5 patients, all possessing the heterozygous variant. The log-rank test showed no difference between the genotypes in the stroke-free survival rate. Furthermore, the p.R4810K genotype was not associated with a poor functional condition. Conclusions: The p.R4810K founder variant of RNF213 affects the phenotype at disease onset. However, the optimal revascularization may be effective, regardless of the genotype, even for the homozygous variant, which has been thought to be the most pathogenic. This genotype may not strongly influence the long-term clinical manifestations or poor prognosis in MMD.

ㄷ 2019 S. Karger AG, Basel

\section{Introduction}

Moyamoya disease (MMD) is an idiopathic cerebrovascular disease featuring progressive occlusion of intracranial arteries and development of abnormal vessels 
at the base of the brain, which are referred to as moyamoya vessels [1-3]. MMD is prevalent in East Asia, including Japan, and a susceptibility gene, RNF213, was previously identified $[4,5]$. In these studies, the c.14429G >A (p.R4810K) variant was identified as a founder variant.

Several studies confirmed that homozygosity of this RNF213 founder variant influenced the severity of the clinical disease phenotype [6-8]. A previous Japanese study showed that its homozygosity was significantly associated with early-onset MMD and cerebral infarction at diagnosis [7]. Additionally, in Korean patients, it was highly associated with early-onset MMD, cerebral infarction at diagnosis, and poor prognosis [8]. The authors suggested that the genotype may be a useful biomarker for early-onset or unstable MMD. However, the association between this genotype and long-term clinical manifestations has remained unclear.

The principal goal of this study was to clarify how the p.R4810K founder variant of the RNF213 moyamoya disease susceptibility gene influences the long-term phenotype in Japanese patients with MMD.

\section{Methods}

Standard Protocol Approvals and Patient Consents

We received approval for this study from the Ethics Committee at our university. Written informed consent was given by all patients. The study is compliant with the Declaration of Helsinki and is based on criteria from the Strengthening the Reporting of Observational Studies in Epidemiology study statement.

\section{Patient Selection}

This study is a retrospective analysis. One hundred Japanese patients with moyamoya disease were initially selected for this study. The carrier status of each patient for the p.R4810K founder variant was determined in our hospital between October 2011 and June 2013. All patients were diagnosed based on the criteria of the Japanese Ministry of Health, Labor, and Welfare [9]. Quasi-MMD (moyamoya syndrome) associated with the underlying conditions were not included $[2,9]$.

Two patients with severe hemorrhage were excluded because they could not undergo appropriate revascularization. One patient was excluded because she selected observation rather than surgery. Two patients were excluded because they underwent indirect bypass in our hospital or another hospital, which was not consistent with our standard treatment options. The other 95 patients underwent direct or combined bypass for revascularization as first-line treatment. One of the 95 patients was excluded because he was lost to follow-up at 1 month after the initial revascularization owing to hospital transfer.

Ultimately, this retrospective study included 94 patients (34 males and 60 females). Thirty-eight were less than 20 years of age at onset.

\section{Study Design}

DNA Sequencing and Data Analysis

Genomic DNA was extracted from the patients' peripheral blood. We identified 3 RNF213 p.R4810K founder variant genotypes (GG/GA/AA) by direct sequencing, setting NM_001256071.1 as the reference transcriptional sequence of RNF213 in the GRCh 37 (hg19) coordinate. The genetic data have been partially included in a previously published report regarding rare variant analysis [6].

Clinical Phenotype Analysis According to Genotype at

Disease Onset and Long Term

The different investigators examined the clinical information by retrospectively reviewing the medical records and interviewing the patient in person or via telephone, thereby blinding the results of the genotype in order to reduce bias. The final status of the enrolled patients was determined between April 2016 and December 2017.

First, the association between the genotype of the p.R4810K founder variant of RNF213 and clinical phenotype was investigated. Patient outcome was evaluated using the incidence of perioperative stroke, recurrent stroke after initial revascularization, and final modified Rankin Scale (mRS), which was also calculated by reviewing the medical records and interviewing the patient in person or via telephone [10]. Stroke was defined as symptomatic cerebral infarction and hemorrhage. Perioperative and recurrent stroke were defined as those that occurred within 1 month from revascularizations or after 1 month from revascularization respectively. The follow-up period was defined as a period from initial revascularization to the final observation.

Second, the association between the genotype of the p.R4810K founder variant of RNF213 and the stroke-free survival rate after initial revascularization was analyzed. Also, clinical factors such as age at onset and initial presentation of onset were analyzed. In terms of Suzuki grade, the grade on the severer side at the time when the disease was first identified in our hospital was applied.

Finally, the genotype and the clinical factors related to mRS were analyzed. The final functional condition was evaluated using $\mathrm{mRS}$, dividing the 2 groups (Good: 0-1, Poor: 2-6).

\section{Statistical Analyses}

The Mann-Whitney U test and Kruskal-Wallis tests were used to compare the age at onset among the 3 genotypes. Each categorical variable of the phenotype or long-term result between the 3 genotypes was calculated using the Fisher's exact test. The KaplanMeier method was used to compare the cumulative stroke-free survival rate with the log-rank test between the 3 genotypes or the other clinical factors. For statistical calculation, the commercial statistical software JMP Pro version 13 (SAS Institute, Tokyo, Japan) was used. A $p$ value $<0.05$ was set as significant.

\section{Results}

All of the 94 enrolled patients were followed up with both at our hospital and at collaborating hospitals; the median follow-up period after initial revascularization were 8 years 4 months (100 months, 30-219 months; Table 1). The final follow-up rate was $92.6 \%$ (87/94). Seven patients were not observed between April 2016 and 
Table 1. Genetic and clinical characteristics of the cohort of 94 patients with MMD

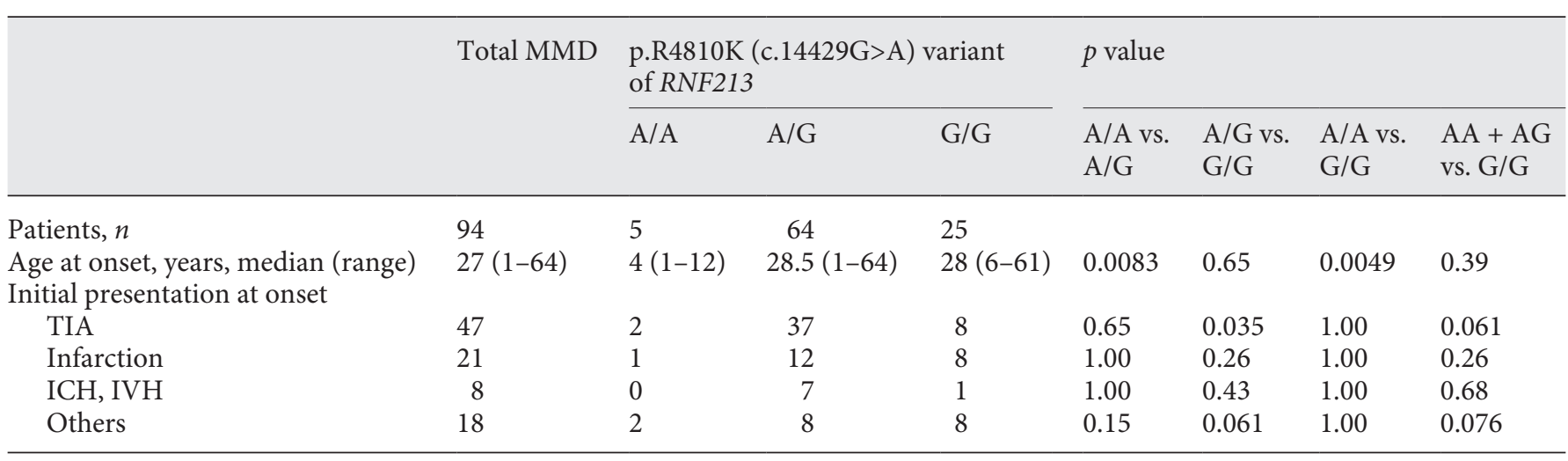

Others included 2 asymptomatic patients and 11 patients complaining of headache.

MMD, moyamoya disease; TIA, transient ischemic attack; ICH, intracerebral hemorrhage; IVH, intraventricular hemorrhage.

December 2017, but they were followed up with later for a median period of 92 months (30-147 months). Therefore, they were all included in the following analyses using data from each final follow-up period.

The p.R4810K (c.14429G>A) Founder Variant of

RNF213 and the Phenotype at the Onset of the 94

Patients with MMD

The p.R4810K (c.14429G>A) variant of RNF213 was detected in $69(73.4 \%)$ of the 94 patients with MMD. The homozygous variant (A/A) and the heterozygous variant $(\mathrm{A} / \mathrm{G})$ were detected in 5 and 64 patients respectively. The median patient age at onset was 27 years (1-64 years). Those with A/A had a median age of 4 years at onset, which was significantly lesser than those with $A / G$ (28.5 years) or G/G (28 years). There was no significant difference in initial presentation at onset between the genotypes except that TIA is slightly associated with $A / G$ compared than $\mathrm{G} / \mathrm{G}$.

\section{Association between the p.R4810K Genotype and} Perioperative Stroke or Recurrent Stroke after Initial

\section{Revascularization}

Among the 94 patients with MMD, perioperative stroke occurred in 6 patients. There was no significant difference among these genotypes in terms of perioperative stroke occurrence (Table 2).

Recurrent stroke occurred in 6 patients $(6.4 \%)$ out of the 94 total patients with MMD. The annual risk of recurrent stroke was $0.72 \%$. It occurred in none of the 5 patients having the homozygous variant, 5 out of 64 with the heterozygous variant, and 1 out of 25 in the wild-type group. There was no significant difference in recurrent stroke among the genotypes. Recurrent cerebral hemorrhage occurred in 5 patients, and they all had the heterozygous variant. One patient with the heterozygous variant experienced both recurrent cerebral infarction and hemorrhage at different phases. With regard to additional revascularization during the clinical course, one patient with the wild-type genotype experienced recurrent transient ischemic attack (TIA) owing to the progression of posterior cerebral artery stenosis and underwent additional revascularization using the occipital artery.

\section{Association between the p.R4810K Genotype}

and Stroke-Free Survival Rate after Initial

Revascularization

The Kaplan-Meier method and the log-rank test did not reveal a significant difference in stroke-free survival rate after initial revascularization between the 3 genotypes ( $p=0.53$, Table 3 , online suppl. Fig. 1 , see www. karger.com/doi/10.1159/000499699). Moreover, age, initial presentation (ischemic or hemorrhagic type) at onset, and Suzuki grade $\geq 4$ did not significantly influence the stroke-free survival rate (Table 3 ).

\section{Genetic and Clinical Factors Related to Final \\ Functional Condition}

The p.R4810K variant genotype was not associated with poor functional condition. Only infarction at onset ( $p=0.0077)$, perioperative stroke $(p=0.0001)$, and recurrent stroke $(p=0.03)$ were significantly associated with it (Table 4). 
Table 2. Association of the p.R4810K founder variant of RNF213 with perioperative stroke and recurrent stroke after revascularization

\begin{tabular}{|c|c|c|c|c|c|c|c|c|}
\hline & \multirow[t]{2}{*}{$\begin{array}{l}\text { Total MMD } \\
(n=94), \%\end{array}$} & \multicolumn{3}{|c|}{$\begin{array}{l}\text { p.R } 4810 \mathrm{~K}(\mathrm{c} .14429 \mathrm{G}>\mathrm{A}) \text { variant } \\
\text { of } R N F 213\end{array}$} & \multicolumn{4}{|c|}{$p$ value OR } \\
\hline & & $\begin{array}{l}\mathrm{A} / \mathrm{A} \\
(n=5), \%\end{array}$ & $\begin{array}{l}\mathrm{A} / \mathrm{G} \\
(n=64), \%\end{array}$ & $\begin{array}{l}\mathrm{G} / \mathrm{G} \\
(n=25), \%\end{array}$ & $\begin{array}{l}\text { A/A vs. } \\
\text { A/G }\end{array}$ & $\begin{array}{l}\text { A/G vs. } \\
\text { G/G }\end{array}$ & $\begin{array}{l}\text { A/A vs. } \\
\text { G/G }\end{array}$ & $\begin{array}{l}\mathrm{A} / \mathrm{A}+\mathrm{A} / \mathrm{G} \\
\text { vs. } \mathrm{G} / \mathrm{G}\end{array}$ \\
\hline \multirow[t]{2}{*}{ Perioperative stroke/perperson } & $6(6.4)$ & $1(20.0)$ & $3(4.7)$ & $2(7.7)$ & \multirow{4}{*}{$\begin{array}{l}0.27 \\
5.08\end{array}$} & \multirow{4}{*}{$\begin{array}{l}0.62 \\
0.57\end{array}$} & \multirow{4}{*}{$\begin{array}{l}0.43 \\
2.88\end{array}$} & \multirow{4}{*}{$\begin{array}{l}0.65 \\
0.71\end{array}$} \\
\hline & & & & & & & & \\
\hline Cerebral Infarction & $3(3.2)$ & $1(20.0)$ & $1(1.6)$ & $1(4.0)$ & & & & \\
\hline Cerebral hemorrhage & $3(3.2)$ & $0(0)$ & $2(3.1)$ & $1(4.0)$ & & & & \\
\hline \multirow{4}{*}{$\begin{array}{l}\text { Recurrent stroke after } \\
\text { revascularization } \\
\text { Cerebral infarction } \\
\text { Cerebral hemorrhage }\end{array}$} & $6(6.4)$ & $0(0)$ & $5(7.8)$ & $1(4.0)$ & \multirow{4}{*}{$\begin{array}{l}1.00 \\
0\end{array}$} & \multirow{4}{*}{$\begin{array}{l}1.00 \\
2.03\end{array}$} & \multirow{4}{*}{$\begin{array}{l}1.00 \\
0\end{array}$} & \multirow{4}{*}{$\begin{array}{l}1.00 \\
1.88\end{array}$} \\
\hline & & & & & & & & \\
\hline & $2(2.1)$ & $0(0)$ & $1(1.6)$ & $1(4.0)$ & & & & \\
\hline & $5(5.3)$ & $0(0)$ & $5(7.8)$ & $0(0)$ & & & & \\
\hline
\end{tabular}

The perioperative stroke number was counted per person, not per surgery. One patient in the A/G group experienced both recurrent cerebral infarction and hemorrhage at different times.

MMD, moyamoya disease.

Table 3. Stroke-free rate after initial revascularization for the p.R4810K founder variant of RNF213 and other clinical factors

\begin{tabular}{|c|c|c|c|}
\hline & $n$ & $\begin{array}{l}\text { Median } \\
\text { stroke-free } \\
\text { period, } \\
\text { months }\end{array}$ & $p$ value \\
\hline \multicolumn{4}{|l|}{ Genotype of the p.R4810K } \\
\hline founder variant & & & $0.53^{*}$ \\
\hline Homozygous (AA) & 5 & 147 & \\
\hline Heterozygous (AG) & 64 & 94.5 & \\
\hline Wild type (GG) & 25 & 115 & \\
\hline Age, years & & & 0.60 \\
\hline$\leq 5$ & 11 & 77 & \\
\hline$>5$ & 83 & 100 & \\
\hline Initial presentation at onset & & & 0.38 \\
\hline TIA & 47 & 92 & \\
\hline Infarction & 21 & 115 & \\
\hline $\mathrm{ICH}, \mathrm{IVH}$ & 8 & 87.5 & \\
\hline Others & 18 & 109.5 & \\
\hline Suzuki grade & & & 0.07 \\
\hline$\geq 4$ & 33 & & \\
\hline$\leq 3$ & 61 & & \\
\hline
\end{tabular}

The log-rank test was used. * See online supplementary Fig. 1.

TIA, transient ischemic attack; ICH, intracerebral hemorrhage; IVH, intraventricular hemorrhage.

\section{Discussion}

\section{Relationship between the RNF213 Genotype and}

Long-Term Clinical Manifestations

To our knowledge, this is the first report demonstrating an association between the RNF213 genotype and long-term manifestations. Information about the genotype-phenotype relationship is important for the future management of $\mathrm{MMD}$, but reports regarding the association between long-term manifestations and the p.R4810K founder variant of RNF213 are scarce. Some reports suggested that the p.R4810K founder variant may be a prognostic biomarker of MMD, but the definition of the long-term outcome used in their studies was unclear $[7,8]$. Whether the R4810K genotype influences the long-term clinical course after revascularization has thus far remained unknown. Over the course of the 8-year mean follow-up period used in our study, the frequency of recurrent stroke was low, regardless of the genotype, and there was no significant difference in recurrent stroke or final mRS value between the genotypes of the p.R4810K founder variant, even in those with the homozygous variant, which would theoretically have the strongest genetic effect. In other words, our result revealed that optimal bypass surgery may be an effective treatment even for the homozygous variant. Interestingly, the patients with the homozygous p.R4810K variant were stable without increased likelihood of recurrent stroke after revascularization. According to our data, it is difficult to predict prognosis after revascularization using the p.R $4810 \mathrm{~K}$ founder variant as a prognostic biomarker, though the homozygous founder variant may influence some aspects of the disease, such as the age of onset, as suggested by prior reports $[6,7]$.

During our long-term observation, recurrent stroke after direct or combined revascularization was observed in 6 cases out of 94 patients (annual risk: 0.72\%). In par- 
Table 4. Genotypic and clinical factors related to the $\mathrm{mRS}$ at the final evaluation

\begin{tabular}{|c|c|c|c|}
\hline & Good (mRS 0-1) 81 & Poor (mRS 2-6) 13 & $p$ value \\
\hline \multicolumn{4}{|c|}{ Genotype of the p.R $4810 \mathrm{~K}$ founder variant } \\
\hline Homozygous (AA) & 4 & 1 & \\
\hline Heterozygous (AG) & 58 & 6 & \\
\hline Wild type (GG) & 19 & 6 & \\
\hline Dominant (AA + AG vs. GG) & & & 0.10 \\
\hline Recessive (AA vs. AG + GG) & & & 0.53 \\
\hline \multicolumn{4}{|l|}{ Age, years } \\
\hline$\leq 5$ & 9 & 2 & 0.65 \\
\hline \multicolumn{4}{|l|}{ Initial presentation at onset } \\
\hline TIA & 43 & 4 & 0.23 \\
\hline Infarction & 14 & 7 & 0.0077 \\
\hline ICH, IVH & 6 & 2 & 0.31 \\
\hline \multicolumn{4}{|l|}{ Suzuki grade } \\
\hline$\geq 4$ & 28 & 5 & 0.76 \\
\hline Perioperative stroke & 1 & 5 & 0.0001 \\
\hline Recurrent stroke & 3 & 3 & 0.03 \\
\hline
\end{tabular}

mRS, modified Rankin Scale; TIA, transient ischemic attack; ICH, intracerebral hemorrhage; IVH, intraventricular hemorrhage.

ticular, recurrent hemorrhage was observed in 5 patients. In several past large studies, the incidence of recurrent stroke after revascularization was reported to be approximately 5-20\% (annual risk: $0.4-6.7 \%$ ) [11-16]. It is difficult to directly compare these incidences because the included patients' characteristics, follow-up rate, or management made a difference to the incidence of recurrent stroke in our study. However, the risk of recurrent stroke, especially hemorrhage, remained even after optimal revascularization. Funaki et al. [17] suggested that this may be due to burden of the moyamoya vessels, a fragile collateral vessel and occurrence of microaneurysm in their cases. In our study, all recurrent hemorrhagic strokes occurred in patients who were heterozygous for the p.R4810K variant, though none occurred in those having the homozygous p.R4810K variant. However, the association could not be confirmed because of the infrequency of recurrent stroke occurrence and the small number of homozygous patients included in our study. Future studies will be needed to further investigate the factors associated with recurrent hemorrhage.

\section{Genetic Issues for RNF213}

The p.R4810K founder variant, which $80 \%$ of Japanese patients with MMD possess, influences long-term clinical course less than expected. This may be because this variant is not located within the functional domains (2 AAA-
ATPase domains and a RING domain) in RNF213, of which the function or the role is not completely analyzed yet. Moreover, rare variants other than the p.R4810K variant were also found in Asian and European patients with MMD $[4,6,18,19]$. Some of those variants appear to be more pathogenic than the $\mathrm{p} . \mathrm{R} 4810 \mathrm{~K}$ founder variant in in-silico prediction tools, such as PolyPhen or C-score of Combined Annotation Dependent Depletion [20-22]. We will have to investigate these rare variants, an unknown gene, or the other factors to establish prognostic markers.

\section{Limitations}

There are some limitations to our study. First, our study did not include many clinical factors showing preoperative severity, such as cerebral blood flow parameters. Although we agree that these clinical factors are important considerations for long-term investigation, we incorporated only the age at onset initial presentation of onset and the Suzuki stage in order to focus exclusively on genetic issues. In our study, the genotype did not have a significant effect on the long-term outcome. This negative correlation between the genotype and long-term outcome would be acceptable as preliminary data for future multifactorial studies incorporating unknown clinical factors influencing the outcomes. Second, this study cannot explain the prognosis of patients who underwent indirect bypass or conservative therapy because this 
study's participants were limited to patients who underwent direct or combined bypass, which is the first line of surgery in our hospital. Furthermore, the rarity and genetic characteristics of MMD influenced the small sample size of patients with the homozygous variant (A/A). Therefore, a prospective study including a larger population is needed.

\section{Conclusions}

This study is the first to demonstrate an association between the genotype of the p.R4810K founder variant of RNF213 and the long-term clinical manifestations in moyamoya disease. Although our study revealed that the p.R $4810 \mathrm{~K}$ variant of RNF213 influences the phenotype at onset, there were not significant differences between this variant and poor prognosis after revascularization. The frequency of recurrent stroke was low, regardless of the genotype, even in patients with the homozygous p.R4810K variant, which has been thought to be most pathogenic; thus, optimal direct or combined bypass surgery may be an effective treatment for all genotypes, even for the homozygous variant.

\section{Acknowledgments}

The authors are grateful to Kazutoshi Hashimoto, MD, Yuichi Mochizuki, MD, Hideaki Onda, MD, PhD, Yasutoshi Kuroi, MD, and Hidetoshi Kasuya, MD, PhD for data sampling.

\section{Disclosure Statement}

The authors report no conflicts of interest regarding the materials in this study.

\section{Funding Sources}

This study did not receive any funding.

\section{References}

1 Kuroda S, Houkin K. Moyamoya disease: current concepts and future perspectives. Lancet Neurol. 2008 Nov;7(11):1056-66.

2 Scott RM, Smith ER. Moyamoya disease and moyamoya syndrome. N Engl J Med. 2009 Mar;360(12):1226-37.

3 Suzuki J, Takaku A. Cerebrovascular "moyamoya" disease. Disease showing abnormal net-like vessels in base of brain. Arch Neurol. 1969 Mar;20(3):288-99.

4 Kamada F, Aoki Y, Narisawa A, Abe Y, Komatsuzaki S, Kikuchi A, et al. A genome-wide association study identifies RNF213 as the first Moyamoya disease gene. J Hum Genet. 2011 Jan;56(1):34-40.

5 Liu W, Morito D, Takashima S, Mineharu Y, Kobayashi H, Hitomi T, et al. Identification of RNF213 as a susceptibility gene for moyamoya disease and its possible role in vascular development. PLoS One. 2011;6(7): e22542.

6 Moteki Y, Onda H, Kasuya H, Yoneyama T, Okada Y, Hirota K, et al. Systematic Validation of RNF213 Coding Variants in Japanese Patients With Moyamoya Disease. J Am Heart Assoc. 2015 May;4(5): 4.

7 Miyatake S, Miyake N, Touho H, NishimuraTadaki A, Kondo Y, Okada I, et al. Homozygous c.14576G[\{GT\}]A variant of RNF213 predicts early-onset and severe form of moyamoya disease. Neurology. 2012 Mar;78(11) $803-10$
8 Kim EH, Yum MS, Ra YS, Park JB, Ahn JS, Kim GH, et al. Importance of RNF213 polymorphism on clinical features and long-term outcome in moyamoya disease. J Neurosurg. 2016 May; 124(5):1221-7.

9 Research Committee on the Pathology and Treatment of Spontaneous Occlusion of the Circle of Willis; Health Labour Sciences Research Grant for Research on Measures for Infractable Diseases. guidelines for diagnosis and treatment of moyamoya disease (spontaneous occlusion of the circle of Willis). Neurol Med Chir (Tokyo). 2012;52(5): 245-66.

10 Savio K, Pietra GL, Oddone E, Reggiani M, Leone MA. Reliability of the modified Rankin Scale applied by telephone. Neurol Int. 2013 Feb;5(1):e2.

11 Starke RM, Komotar RJ, Hickman ZL, Paz YE, Pugliese AG, Otten ML, et al. Clinical features, surgical treatment, and long-term outcome in adult patients with moyamoya disease. Clinical article. J Neurosurg. 2009 Nov; 111(5):936-42.

12 Kim T, Oh CW, Kwon OK, Hwang G, Kim JE, Kang HS, et al. Stroke prevention by direct revascularization for patients with adultonset moyamoya disease presenting with ischemia. J Neurosurg. 2016 Jun;124(6): 1788-93.

13 Kuroda S, Houkin K, Ishikawa T, Nakayama N, Iwasaki Y. Novel bypass surgery for moyamoya disease using pericranial flap: its im- pacts on cerebral hemodynamics and longterm outcome. Neurosurgery. 2010 Jun;66(6): 1093-101.

14 Guzman R, Lee M, Achrol A, Bell-Stephens T, Kelly M, Do HM, et al. Clinical outcome after 450 revascularization procedures for moyamoya disease. Clinical article. J Neurosurg. 2009 Nov;111(5):927-35.

15 Funaki T, Takahashi JC, Takagi Y, Yoshida K, Araki Y, Kikuchi T, et al. Incidence of late cerebrovascular events after direct bypass among children with moyamoya disease: a descriptive longitudinal study at a single center. Acta Neurochir (Wien). 2014 Mar;156(3):551-9.

16 Liu XJ, Zhang D, Wang S, Zhao YL, Teo $\mathrm{M}$, Wang $\mathrm{R}$, et al. Clinical features and long- term outcomes of moyamoya disease: a single-center experience with 528 cases in China. J Neurosurg. 2015 Feb;122(2): 392-9.

17 Funaki T, Takahashi JC, Yoshida K, Takagi Y, Fushimi Y, Kikuchi T, et al. Periventricular anastomosis in moyamoya disease: detecting fragile collateral vessels with MR angiography. J Neurosurg. 2016 Jun;124(6): 1766-72.

18 Guey S, Kraemer M, Hervé D, Ludwig T, Kossorotoff M, Bergametti F, et al.; FREX consortium. Rare RNF213 variants in the C-terminal region encompassing the RING-finger domain are associated with moyamoya angiopathy in Caucasians. Eur J Hum Genet. 2017 Aug;25(8):995-1003. 
19 Kobayashi H, Brozman M, Kyselová K, Viszlayová $\mathrm{D}$, Morimoto $\mathrm{T}$, Roubec $\mathrm{M}$, et al. RNF213 Rare Variants in Slovakian and Czech Moyamoya Disease Patients. PLoS One. 2016 Oct;11(10):e0164759.
20 Adzhubei IA, Schmidt S, Peshkin L, Ramensky VE, Gerasimova A, Bork P, et al. A method and server for predicting damaging missense mutations. Nat Methods. 2010 Apr; 7(4):248-9.

21 Kircher M, Witten DM, Jain P, O’Roak BJ, Cooper GM, Shendure J. A general framework for estimating the relative pathogenicity of human genetic variants. Nat Genet. 2014 Mar;46(3):310-5.
22 Nomura S, Kawashima A, Akagawa H, Kawamata T. Letter to the Editor. Influence of rare RNF213 variants other than p.R4810K on the clinical outcomes of moyamoya disease. J Neurosurg. 2018 Aug;129(2):563-5. 